\title{
Hyperdimensional Perspectives in Out-of-Body and Near-Death Experiences
}

\author{
Robert J. Brumblay, M.D., F.A.C.E.P. \\ Honolulu, HI
}

\begin{abstract}
Recent theories of modern physics predict that the universe has more dimensions than are apparent to us. Many near-death experiencers report the perception that there are more dimensions than we are commonly aware of. These two statements might be related. This article examines the possibility of additional dimensions (hyperdimensions), what they would seem like, and whether they seem to be described by the unusual visual perspectives found in out-of-body and near-death experience accounts. I examine some implications of a hyperdimensional model of the universe.
\end{abstract}

KEY WORDS: near-death experience; out-of-body experience; dimension; time; hyperdimensional; hyperspatial; perspective.

A "miracle" is commonly considered to be an effect or event without law, or beyond law. But all events in our precisely adjusted universe are lawtully wrought and lawfully explicable.

Paramahansa Yogananda (1946, p. 321, footnote)

Published accounts of near-death experiences can include descriptions of the ability to see through walls. This is one of the most striking examples of how these experiences seem to violate the laws of nature. Recent theories of physics such as superstring theory predict the existence of more dimensions than we commonly perceive. A specific example is M-theory, which builds on the concept of superstring theory and includes ten spatial dimensions, with time as an eleventh (Greene,

Robert J. Brumblay, M.D., F.A.C.E.P., is an emergency physician and Medical Director for the Emergency Services Department, City and County of Honolulu, HI. This article was based in part on a presentation to the Annual Conference of the International Association for Near-Death Studies, Seattle, WA, 2001. Reprint requests may be addressed to Dr. Brumblay at 105 Nakeke Place, Wahiawa, HI 96786; e-mail: brumblay@hawaii.rr.com. 
1999). The commonly accepted scientific view over the last 20 years is that these dimensions do in fact exist, but are not experienced by us, and are therefore somehow made inapparent by being tightly convoluted into the fabric of space. More recently (Arkani-Hamed, Dimopoulos, and Dvali, 2000) there has been consideration of the possibility that the dimensions could be much larger than previously thought. This would solve some problems in physical theory. Through an analysis of hyperdimensional geometry (Abbott, 1884; Kaku, 1994; Rucker, 1977), it can be demonstrated that if these dimensions do in fact exist, and are large, they would allow the novel visual perspectives described in these near-death experience accounts without violation of any natural laws.

\section{The Experience of Transformation from Lower to Higher Dimensions}

The easiest way to understand the kind of visual perspectives that would be expected with a transition to a higher dimensional environment is to start with simpler models and from them develop models of greater complexity. I will consider first what it would be like to be accustomed to living in a universe of only one dimension, and develop an understanding of what it would be like to be moved to a two-dimensional universe. I will then repeat the process for a being accustomed to living in a two-dimensional universe who is then moved into a space of three dimensions. Finally, I will extend the process to the case of a three-dimensional being who is moved into a space with at least one additional spatial dimension. What is learned from the simpler models can be extrapolated to the more complex case in an understandable manner.

Following the progression of this line of thought may be easier if Table $I$ is referred to as a guide. Certain assumptions must be made during this process in order to make the analysis consistent with the near-death and out-of-body experiences described. These assumptions can be considered corollaries or predictions of this model and will be listed later.

Once we have derived our model defining what kinds of experiences we are looking for, we will review some of the available published accounts of out-of-body and near-death experiences to see how these accounts are consistent with the model, and how they could be interpreted as perceptions of higher dimensional phenomena. 












\section{Experiencing a Two-Dimensional Universe as a} One-Dimensional Being

What would it be like to be a one-dimensional being living in a onedimensional universe? The being could be any size from a point to a line segment, or even up to the size of the line itself, the limits of the universe. If the being's size were to be finite and limited, there would be room in the linear universe for other beings (Figure 1). Each being would have a clear "line of sight" only as far as the near end of the next being on the line. A wall could be as small as a single point, and still completely obstruct vision and movement. No being could see or move past any other being on the line. None of the beings in this universe would be able to imagine any directions other than the two directions that we will refer to here as right or left on the line, and no such being could imagine or visualize a universe of more than one dimension.

Now let us move a line segment being out of this limited linear universe, into a two-dimensional universe, in this case moving the fellow out into a planar universe (Figure 2). We will leave his physical body behind and instead pull out what we will refer to here as his "astral" body, which for purposes of this discussion we will assume has a structure that generally corresponds to the structure of his physical body. Once a short distance out of his usual position on the line, his astral body would be able to look up and down the line, seeing not only his usual limited view of the near ends of his two nearest neighbors, but also the farther ends and all the interior features in between (Figure 3).

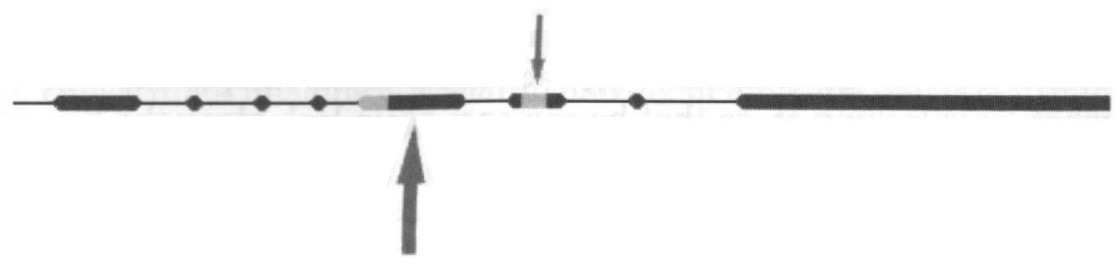

Figure 1. This is a representation of a linear universe populated by entities varying in size from points to line segments. In order to be visible, the universe and entities pictured here are shown with thickness that they would not actually have if they were truly one-dimensional. If we consider what it would be like to be the being indicated by the large arrow, our perception of the adjacent being (small arrow) would be limited to the near end of that line segment. We would not be able to tell if this being was a point or a line segment. We would not be able to "see" the far end of that adjacent being or the light gray internal feature shown in this picture. We would only be aware of our two immediate neighbors and would have no ability to perceive any part of the linear universe beyond them. 


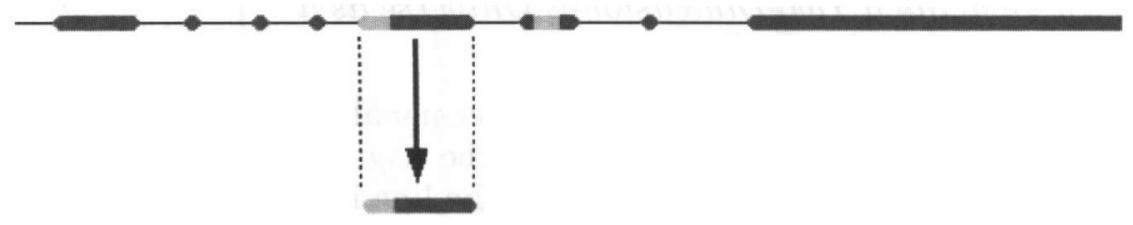

Figure 2. Here we separate what we will call the "astral body" from the "physical body" of the line segment entity we are considering. The physical body remains on the line, in the original one-dimensional space. The astral body is shown with a structure that somehow corresponds to that of the physical body, but is now in the larger, planar space of the paper on which this image is printed.

Because he can only mentally visualize movement in two directions, and it is clear to him that he has not moved right or left on the line, he still mentally visualizes himself in the same position on the line. To him, the neighbors on the line have become transparent, and he feels that he is looking through them, not around them. If he moves right or left closely parallel to the line, it seems to him as if he has moved through his neighbors or any nearby walls. If he moves away from the line in a perpendicular direction, the neighbors appear more faint, or as if they are dissolving, but they do not seem to grow more distant because he is neither moving right nor left relative to them.

Without moving too far off the line he can turn and look back at the line, seeing both to the right and the left at the same time (Figure 3). In order for this to occur, his astral vision must be capable of sensory input in at least one more dimension than his normal physical vision. The visual information gathered in this way exceeds the complexity of mental processing to which he is accustomed. He may find himself more comfortable at this point moving back closer to the line, to one side of what he is looking at, so that he can once again look along the line at

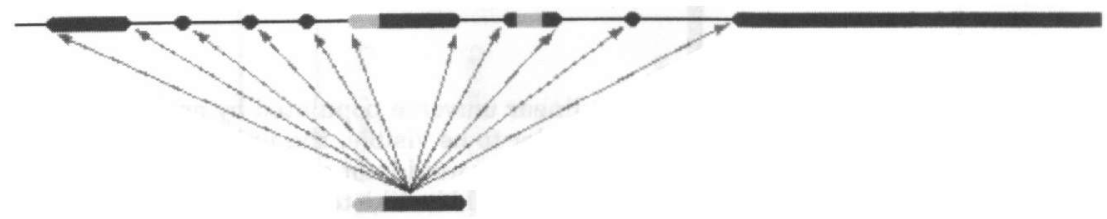

Figure 3. From its new vantage point in a higher dimensional space of two dimensions, the line segment now has "line of sight" accessibility to all points and structures of the linear universe. Now it can see all its neighbors, whether they are points or line segments, and can see their internal structures, including those of its own physical body. 


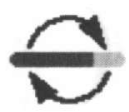

Figure 4. The displaced astral body of the line segment can now rotate in the higher dimensional space of two dimensions, a type of movement that would have been impossible to do or even to imagine for a being remaining in the original one-dimensional universe. If moved back toward its physical body it would seem impossibly reversed relative to it.

it. This will provide him with the most understandable visual input of any position off of the line, because the objects he is looking at will be in alignment with him and with each other. Neighbors or walls will be closest to their normal positions relative to what he is familiar with, and they will seem the least transparent.

Once off the line, he can now rotate around in the planar universe 180 degrees and move back toward his physical body remaining on the line (Figure 4). He will now find himself reversed relative to his body in a way that is unimaginable to him based on his usual concepts of geometry.

\section{Experiencing a Three-Dimensional Universe as a Two-Dimensional Being}

Next imagine a planar, two dimensional universe populated by flat, two dimensional beings (Figure 5). Their line of sight only extends to the near, exterior surface of their neighbors (Figure 6). They cannot see the far perimeter of any neighbor, and cannot see interior structures.

They cannot imagine or visualize moving in any direction other than the four we will refer to here as right, left, up, and down. They cannot imagine or visualize any space or universe of more than two dimensions. They can see the far perimeter of a neighbor only by rotating around each other in the plane, and a line segment becomes a wall that completely obstructs vision and movement (Figure 7).

This time let us move the astral body of one of these planar beings out of their planar universe, into a three-dimensional space (Figure 8). Once he is barely out of his usual position on the plane, the being will have a direct line of sight past walls (Figure 9). He will now be able to see the far perimeter of neighbors, and also any interior features. He correctly knows that he has not moved right, left, up, or down from his original position on the plane. He cannot imagine having moved 


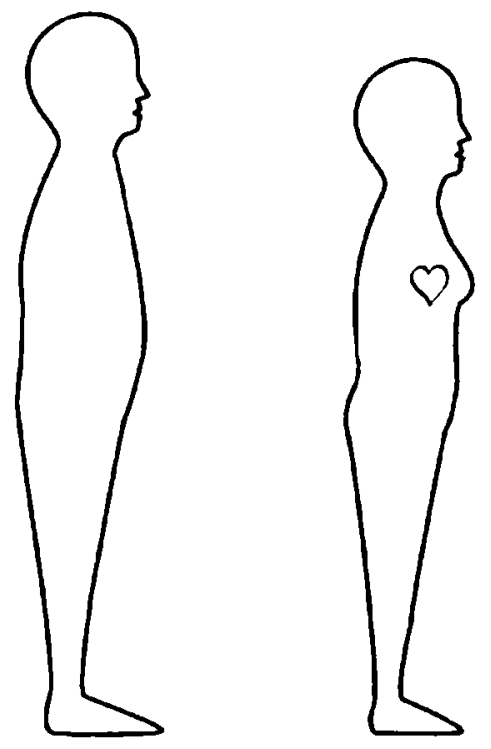

Figure 5. These are representations of two-dimensional beings in a two-dimensional universe. They look like outlines of threedimensional humans, but this appearance was chosen for understandability. Their external surface is the perimeter of each figure.

spatially in any direction other than those listed, so he feels at this point that he is looking through objects and that they are now transparent. If he moves farther from his position on the plane, the objects there will become more transparent or will dissolve out of sight, and he will still be correct in his sense that he has not moved right, left, up, or down.

Without moving too far from the plane he can now rotate in three dimensional space and look back at his planar world (Figure 9). As long as his astral vision perception is of at least one more dimension than he is accustomed to, he will have a 360-degree circular visual field, but this will exceed his normal capability for mental visualization of geometric relationships. If he remains in the center of the room, he can see everything around him in the same view, which will be a perspective difficult for him to interpret. He may feel more comfortable moving back toward the plane, and looking at any features of interest from a position barely off the plane and to the side of those features, as if from the side 


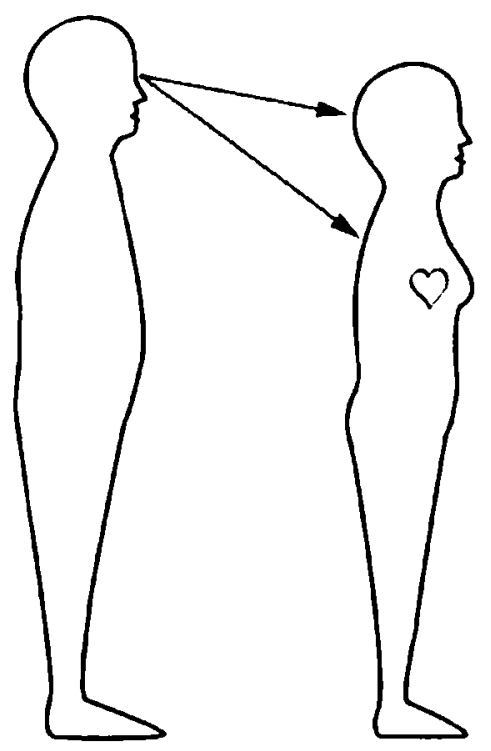

Figure 6. The male figure only has line of sight access to the near surface of the female figure. He can not view her face unless he rotates around her in the plane of the paper, turning so that he is upside down. He can not see any internal structures she may have, here represented by a heart.

of a room, near its wall. From this position, neighbors and walls will seem to be positioned closest to their normal visual relationships and they will be least transparent. Once off the plane, he can now rotate in three dimensional space and flip like a pancake (Figures 10 and 11). If he only flips once and returns to his physical body, he will be reversed relative to it in a manner unimaginable to him or others from his planar world.

\section{Experiencing a Four-or Higher-Dimensional Universe as a Three-Dimensional Being}

By extrapolating from the above discussions about lower dimensional transitions, it is possible to understand what visual perspectives would be expected when moving from a three-dimensional universe to a 


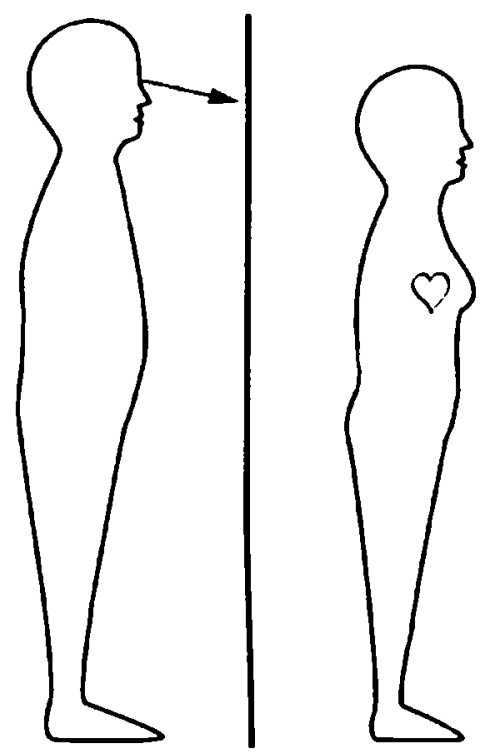

Figure 7. In this planar universe, a line segment forms a wall. The simple line segment blocks both movement and sight.

universe of higher dimensions that we normally cannot imagine or visualize. Please follow the line of thought by referring to Table I.

We start with our three-dimensional being in a world where a wall minimally consists of a plane. He can only see the near surface of a neighbor and not far surfaces or interior features. He can visualize moving only up, down, right, left, forward, or backward. He can imagine a space only of three dimensions. If asked to point in the direction of a fourth spatial dimension, he cannot.

Now we move the astral body of our three-dimensional being away from his physical body, and out into a universe of at least one additional spatial dimension. As soon as he moves out of the volume of his threedimensional world, he gains a direct line of sight past walls. He can now see far surfaces of objects, for total surface vision. He can now see the interior features of closed objects. Yet he has not moved in any direction he is familiar with. To his perspective, he is still in the same place. He has not moved up, down, right, left, forward, or backward. The visual appearance of his world has changed around him, so the walls 


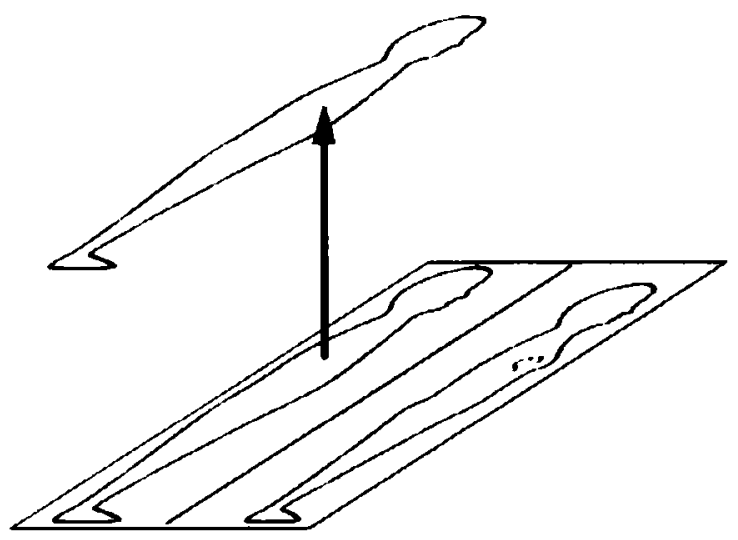

Figure 8. Here we separate the astral body of the male figure from its physical body remaining behind in the planar universe. We are assuming for the sake of this discussion that the astral body has a structure that has general correspondence to the structure of the physical body.

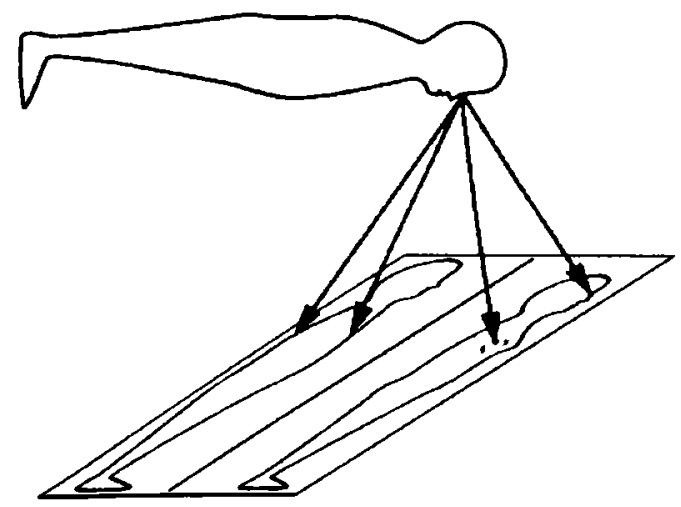

Figure 9. Once removed from the limitations of the planar space, the astral body is now free in threedimensional space to turn and look back at the features of the planar space from a vantage point outside of it. Now it becomes possible to see both the front and back of the male's own physical body simultaneously. The female's physical body can be seen similarly, including internal structures such as the heart depicted here, even though it is located on the other side of the line segment wall. 




Figure 10. By rotating the astral body of the male being around a longitudinal axis, the astral body can be turned so that it is reversed front to back.

appear in the same place as before, but now appear transparent. If he moves farther away in the direction of the additional spatial dimension, the walls will fade without necessarily seeming to have moved farther away.

If he turns and looks back at his world before he moves very far away, he will see the features of the world with 360-degree spherical vision. This will exceed his normal visual interpretation of spatial relationships, and he will be more comfortable moving back as close to his three-dimensional space as he can. This will minimize transparency



Figure 11. By rotating the astral body of the male being around a transverse axis, the astral body can be turned so that it is reversed top to bottom. 
of objects. He will also want to view any scene of interest from outside of that scene, in order to maintain a visual perspective as close to normal as he can. Placing himself anywhere else in the interior of the scene returns him to a 360-degree spherical view, in which he is surrounded by the objects he is trying to observe, and this is likely to be confusing visual input. Further, placing himself against a wall reduces the field of his 360-degree spherical vision to 180-degree hemispherical vision, which is closer to his normal view of things in his three-dimensional world. He still has spherical vision, but the objects of attention are gathered together into a more restricted visual field and are easier to observe. A wall-ceiling corner reduces the field of view still further, which may help more. If an observer could pick any outside perspective to view a scene, a wall-floor corner perspective would in most cases make the least sense, and a wall-ceiling perspective the most. This can be understood by imagining yourself as a movie director: A scene you are filming would be much more understandable if it is shot with the camera overhead rather than pointed upward from the floor.

\section{Example Accounts of Hyperdimensional Imagery}

\section{0-Degree Spherical Vision}

Kenneth Ring and Evelyn Elsaesser Valarino (1998) related an example of 360-degree spherical vision during a near death experience. Please note that in their book, the experiencer quoted erroneously said "three hundred degree" rather than "three hundred sixty degree." This error was confirmed by personal communication with Ring and the quote below is in corrected form:

I was hovering over the stretcher in one of the emergency rooms at the hospital. I glanced down at the stretcher, knew the body wrapped in blankets was mine, and really didn't care. The room was much more interesting than my body. And what a neat perspective. I could see everything. And I do mean everything! I could see the top of the light on the ceiling, and the underside of the stretcher. I could see the tiles on the ceiling and the tiles on the floor, simultaneously: three hundred sixty degree spherical vision. And not just spherical. Detailed! I could see every single hair and the follicle out of which it grew on the head of the nurse standing beside the stretcher. At the time, I knew exactly how many hairs there were to look at. (pp. 6263) 


\section{Total Surface Vision}

Betty Eadie described her total surface vision during her near-death experience as follows:

It was almost as if I felt a pop or release inside me, and my spirit was suddenly drawn out through my chest and pulled upward, as if by a giant magnet.... I was above the bed, hovering near the ceiling... I turned and saw a body lying on the bed. I was curious about who it was, and immediately I began descending toward it.... And then I recognized that it was my own. That was my body on the bed. I wasn't taken aback, and I wasn't frightened; I simply felt a kind of sympathy for it.... I realized that I had never seen myself three-dimensionally before; I had only seen myself in the mirror, which is only a flat surface. But the eyes of the spirit see in more dimensions than the eyes of the mortal body. I saw my body from all directions at once-from in front, behind, and from the sides. I saw aspects to my features I had never known before, adding a wholeness, a completeness to my view. (Eadie and Taylor, 1992, pp. 62-63)

\section{Interior Feature Vision}

An account of interior feature vision during a near-death experience was provided to me by an elderly lady whom I will call Ruth. She had her experience while in an intensive care unit with septic shock. Ruth stated that she found herself drifting out of her room in the intensive care unit, while her physical body remained on the bed. She saw her daughter in the waiting area, and could immediately see that her daughter was pregnant with a little boy. Later, after her daughter delivered the baby and Ruth held him for the first time, she had the feeling they had already met before, during her near-death experience.

\section{Seeing Through Walls}

This description of seeing through walls during a near-death experience was provided to me by a woman who at age 14 suffered a respiratory arrest during an anaphylactoid reaction to intravenous $x$-ray contrast medium. Especially notable is that this experience occurred in an x-ray room with lead-lined walls:

When I woke up I was above my body. I was aware of all the people who had been called into the room to help and what they were thinking. I also found myself able to see my mother on the other side of the wall in the waiting room. She was sitting with her hands in her lap and was crying because she was being told about what had happened to me. At 
the same time I could also see other people in other nearby rooms, all separated from the x-ray area by walls. Someone in another room was getting some kind of physical therapy treatment. But my attention was drawn to my mother. I still could tell that the walls were there, but I could see the other people in the other rooms.

Another example came from an adult female attendee of the programs at the Monroe Institute, who is able to initiate out-of-body experiences essentially at will. She was asked what the visual differences are when moving into the out-of-body state, and whether it makes any difference if her physical eyes are open or closed. She responded that even if she was lying in the dark and her eyes were closed, the room becomes visible as she shifts out of her body. There is a kind of light diffusely present that does not cast shadows. The walls at the same time become transparent and she can see or move beyond them.

\section{Mirror-Image Reversal}

Robert Monroe, the founder of the Monroe Institute, described reversed perception of his own physical body:

After "lifting" out easily, and holding control in the same room, I finally got up the courage to go back and carefully examine the physical body remaining on the bed. I started down slowly, reaching out in the semidarkness.... I reached down carefully to touch my physical head, and my hands touched feet! At first, I thought I had drifted somewhere else, and I felt my toes. My left big toe has a thick nail due to a longago mashing by a dropped log. This big toe (left) did not! I felt with my hands to the right foot. The big toe on the right foot did have the thick nail. Everything was reversed, like a mirror image.... Strange, the reversal. Floating in the half-darkness, I could have swung around and become disoriented. But the thick nail was on the right foot instead of the left. (1971, pp. 172-173)

\section{Multiple Hyperdimensional Characteristics}

The following example has been included because it includes several hyperdimensional characteristics. The quote is from Paramahansa Yogananda's description of his first experience with samadhi, which is a kind of direct experience of God. Samadhi can be attained through certain meditational and yogic techniques. In this case, this state was initiated for the student by an action of his guru, and started out with a sense of expansion of self-identity out of the body. The quote describing 
an initial part of the experience includes interior feature vision, 360degree spherical vision, and seeing through a wall:

The roots of plants and trees appeared through a dim transparency of the soil, I discerned the inward flow of their sap.

The whole vicinity lay bare before me. My ordinary frontal vision was now changed to a vast spherical sight, simultaneously all-perceptive. Through the back of my head I saw men strolling far down Rai Ghat Lane, and noticed also a white cow that was leisurely approaching. When she reached the open ashram gate, I observed her as though with my two physical eyes. After she had passed behind the brick wall of the courtyard, I saw her clearly still. (1946, p. 142)

\section{Adding Time to the System}

Space and time have been considered to be closely related since the theory of relativity was generally accepted. If spatial dimensions are perceived differently during near-death and out-of-body experiences, there is reason to expect that time also will be perceived differently. The simplest example of this is that near-death experiencers frequently make the claim that they felt as if they were "outside of time" during the course of their experience. If you could actually move outside of time, and then turn around and "look back" at it, what could be seen? If time is considered a dimension closely related to the dimensions of space, it can be reasoned that someone who has moved to a hyperdimensional region will have the same kind of expanded overview of time that he has of spatial objects. You might be able to perceive events from different times, either in the past or future. You might even be able to perceive an event that occurred over time all at once, as a single complex object. If you were able to look at your life as a single very complex object, it would certainly be an object that is extremely lumpy or irregular in form.

While events in the past might appear to be fixed, the future must exist as an incompletely fixed form. The future might include a number of different possible courses of events, and these may shift in probability, or in how fixed they are, with changes in vantage point of the observer.

To comprehend viewing the past, present, and future, consider the analogy of watching a leaf going down a stream from above. Imagine that upstream the streambed is fixed, but downstream the stream spreads out over a flat, sandy delta. As the leaf travels downstream it cannot tell where it is going to go, but from your vantage point, you can see the stream shifting back and forth as it meanders on the sandy 
delta. You can see the various probable courses for the leaf. You could intervene by changing the course of the leaf, either by reaching into the stream with a paddle and deflecting the water ahead of the leaf, or by reaching into the water slightly behind the leaf and stroking with the paddle to change the course of the leaf. The first case is analogous to changing the future of the leaf by altering the future directly. The second case is like changing the future course of the leaf by altering a past event.

In the same way that certain aspects of near-death experiences are perceived by metaphorical means, we should accept that there may be metaphorical means of perception of different times. For example, the decision whether or not to return to life during a near-death experience is sometimes associated with a physical representation of a limit beyond which there can be no return. In his first book on near-death experiences, Raymond Moody noted that this limit may appear as "a body of water, a gray mist, a door, a fence across a field, or simply a line" $(1975$, p. 73$)$. All of these appear to be metaphorical representations of a decision point, beyond which there is no return to life. A metaphor allowing perception of the past or future, such as a television screen or cinematic projection, would be consistent with what we have learned from near-death experiences.

\section{Perception of Events from the Past}

George Ritchie described perceiving past events during his neardeath experience as follows:

Here stood a being that knew everything I had ever done in my life, for the panorama of my life surrounded us, and yet He totally accepted and loved me.... I could see my birth and the death of my own mother a month later. . . A short time later, I could see my sister, Mary Jane, who was almost three years older than I, along with Miss Williams, and my grandparents and father, looking at me in the crib. (1998, pp. 34-35)

\section{Perception of Events from the Future}

Ned Dougherty described perceiving future events during his neardeath experience as follows:

The Lady of Light waved her right arm, and I watched as scenes, as if in a movie, developed before me. I became aware that I was watching future events of my life, scenes that were incongruent with the life that I had been living so far. $(2001$, p. 74$)$ 


\section{Perception of Events Over Time as a Single Complex Object}

Ring and Valarino presented an example of perception during a neardeath experience of events occurring over time as a single complex object:

With regard to the question of time, everything happened instantaneously. The whole thing happened all at once but we are bound by the restraints of language... It is like an explosion, it is all there. When my life went before my eyes, it was from my earliest memory at thirteen months. There was an enormous TV screen in front of me.... Way over on the left was my memory at thirteen months, and way over on the right was July, 1972, age thirty-eight. Everything in between was right there and I could see the whole thing, all at the same instant. (1998, p. 150)

\section{The Hyperdimensional Model of Divine Intervention}

According to this model, any being in higher-dimensional space would be able to observe beings in any lower-dimensional region, without generally being perceived by them. Those beings and their surroundings would be seen from a visual perspective less limited than that available to the lower-dimensional beings themselves. For example, interior objects, such as the internal organs of the lower-dimensional beings, would be clearly visible. There would be no spatial restriction preventing the higher-dimensional being from reaching into the lower-dimensional region and directly affecting objects there, even internal objects or organs (Figure 12). In addition, the higherdimensional being would be able to see, from his or her perspective, the way future events would be altered, even as the intervention is performed.

\section{Discussion}

Available accounts of near-death and out-of-body experiences occasionally include remarkable visual perspectives that are consistent with a hyperdimensional model of perception. Although most published neardeath experiences do not describe this kind of perspective, the existence of such visual perspectives in at least some accounts may be important. Why should hyperdimensional perspectives be produced by the mind without an external objective hyperdimensional experience? On the other hand, the existence of this imagery does not prove that additional dimensions exist. 


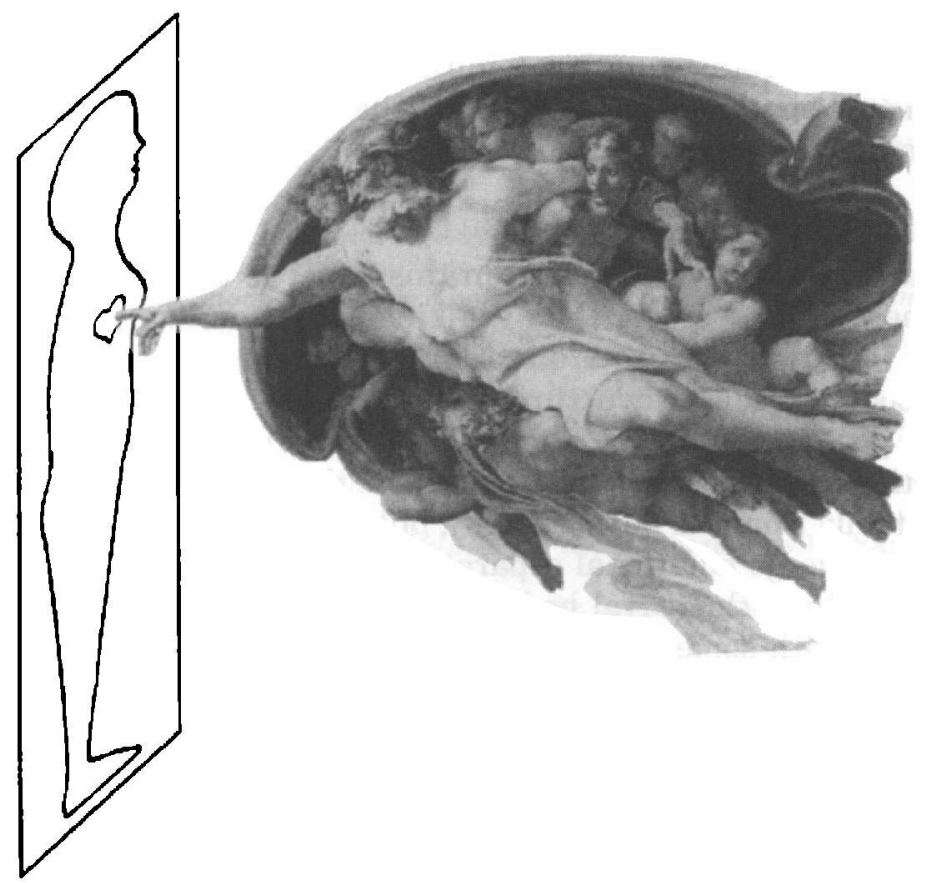

Figure 12. A depiction of divine intervention by a three-dimensional being (the image of God, from The Creation of Man, by Michelangelo, painted 1511-1512, the Sistine Chapel, Vatican City) who is able to view and interact directly with a lower dimensional being including with its internal structures. At the same time, the higher-dimensional being is not within the planar universe, and so can not be seen by the female being or others in that limited universe.

The hyperdimensional model was elaborated with certain assumptions that were found to be necessary. If these corollaries can be tested and proven false, the model as outlined here can be proven false. These corollaries include the following:

1. When a person has an out-of-body experience, there is an aspect of the person that is able to retain a kind of physical form that we are referring to for purposes of this discussion as an "astral body."

2. The astral body has the capability of perception at a distance, and the concept of "line of sight" has a functional meaning when applied to this kind of perception.

3. The astral body perception at a distance has a higher dimensional capability of imaging objects than the visual system of the original being. 
4. The astral form, while able to move in an additional spatial dimension, retains at least initially the limited mental processing of spatial relationships of the original lower-dimensional being.

If the "astral body" described in this paper is the same as the consciousness of the person, then the kinds of vision-like perception described in this paper are a direct perception by consciousness. If the astral body includes a structure that interfaces between consciousness and the perceived universe, then there is a more indirect mechanism of perception. Some religious traditions hold that consciousness can separate not only from the physical body, but also from the astral body, and possibly undergo additional separations. In any case, whether what I am here calling the astral body represents consciousness alone or is associated with some other structure, the perceptions described here tend to have occurred early in the separation stage of near-death and out-of-body experiences. Because of this, these perceptions may represent an interaction of the hyperdimensional astral body with the more limited dimensional structure of our world, a special case of perception that would not continue after the experiencer moves farther away from our three-dimensional physical world.

At this point, it is appropriate to comment on an analysis of visual perception during the near-death experiences of the blind, done by Ring and Sharon Cooper (1999). Their report of a series of near-death experiences of blind people showed that most had some kind of vision-like perception during their experience. Because the perception was clearly not associated with any function by the physical eyes, they suggested that the perception was by a mechanism that they call "transcendental awareness." The possible mechanisms of perception suggested in the preceding paragraph could be considered additional characterization of the same mechanism proposed by Ring and Cooper.

The hyperdimensional model provides us with a theoretical means to advance our definition and understanding of near-death experiences. Once this model is understood, some of those aspects of these experiences that heretofore have been regarded as ineffable may become describable and understandable. If the additional dimensions predicted by modern physical theories are accepted without limitation of their size, then this model allows an explanation of the events described in these experiences that does not violate the laws of nature. 


\section{References}

Abbott, E. A. (1884). Flatland: A romance of many dimensions. New York, NY: Dover.

Arkani-Hamed, N., Dimopoulos, S., and Dvali, G. (2000). The universe's unseen dimensions. Scientific American, 284(8), 62-69.

Dougherty, N. (2001). Fast lane to heaven. Charlottesville, VA: Hampton Roads.

Eadie, B. J., and Taylor, C. (1992). Embraced by the light. Placerville, CA: Gold Leaf Press.

Greene, B. (1999). The elegant universe. New York, NY: Vintage Books.

Kaku, M. (1994). Hyperspace: A scientific odyssey through parallel universes, time warps and the tenth dimension. New York, NY: Doubleday.

Monroe, R. A. (1971). Journeys out of the body. New York, NY: Doubleday.

Moody, R. A. (1975). Life after life. Covington, GA: Mockingbird Books.

Ring, K., and Cooper, S. (1999). Mindsight: Near-death and out-of-body experiences in the blind. Palo Alto, CA: William James Center for Consciousness Studies.

Ring, K., and Valarino, E. E. (1998). Lessons from the light. New York, NY: Plenum/ Insight.

Ritchie, G. G. (1998). Ordered to return: My life after dying. Charlottesville, VA: Hampton Roads.

Rucker, R. V. B. (1977). Geometry, relativity, and the fourth dimension. New York, NY: Dover.

Yogananda, P. (1946). Autobiography of a yogi. Los Angeles, CA: Self-Realization Fellowship. 